\section{Briginal \$rticleg.}

\section{A REVIEW OF RECENT EXPERIMENTAL WORK ON HEMORRHAGIC CONDITIONS.*}

By William palmer luCAs, M.D., Boston.

IN discussing the problem of hemorrhagic diseases in children it is in place first of all to go into the theories underlying these conditions. Of prime importance is the question of what changes are to be found in such conditions, and whether the changes are in the tissues in general, in the blood vessels or in the blood itself. Secondly, has experimental work given any clue to etiology or to treatment, and, if so, do these fit in with the clinical pictures as seen by the general practitioner?

In considering the blood we are at present chiefly interested in its power of coagulation when taken into a suitable vessel. For it has been shown that, if blood is drawn off through a canula coated with a smooth layer of paraffin into a vessel also lined with this substance, the blood will not coagulate; it will remain for hours in the same liquid condition in which it is found in the blood vessels. Now, if a glass bead or rod is dropped into the blood, it will take but a few minutes before it has changed its form entirely and become coagulated. The factors necessary for this coagulation must all have been present before, though unable to act. The exact steps in this complex are a combination, first, of the three precursors of fibrin ferment, the prothrombin, the thrombokinase and the calcium salts, uniting to form the true thrombin which does not exist in the circulating blood except potentially in the form of its precursors. The thrombin thus formed unites with the fibrinogen which is present as such in the circulating blood to form the fibrin in the blood clot. When the substances are present in their proper proportions then we have a normal firm clot, but with the absence of any one of these, the form of the clot is either materially changed or the blood remains fluid, being unable to clot. If, for instance, the salts are diminished, then the prothrombin uniting with the thrombokinase forms a clot that has a greatly delayed coagulation time. If the salts are present and the prothrombin is absent, then the time element is not changed, but the form of the clot is materially altered, and, instead of getting a firm fibrin clot which in a short time contracts and becomes surrounded by a clear serum, we get a homogeneous gelatinous coagulum; in other words, with the loss of the prothrombin, the contractility of the clot is almost lost without affecting the coagulation time. If, now, we suppose the thrombokinase to be lacking, then both the time element and the form of the clot are changed, the blood in these instances acting very much like the blood drawn into the paraffin dish.

The possible changes in the blood vessels that might. lead to hemorrhage are, first, friability, a condition most prone to.occur at the two ex-

\footnotetext{
* Read before the New England Pediatric Society, at Providence,
} R. I., May 8, 1909 . tremes of life; and, second, some change in the lining of the blood vessels conducive to a more easy escape of blood so that osmosis might be freer than normal; or a change in the size of the vessels of such extent that osmosis is brought about by the increased blood pressure. That some of these conditions do occur we shall see in discussing the clinical forms. Lastly, change in the body cells that might chemically alter the normal processes of coagulation by either adding an incompatible substance or by the withdrawal of some substance that is needed in the process of the normal coagulation.

Before bringing forward the experimental work it will be well to look into the accepted etiological theories concerning hemorrhagic conditions. Hemorrhage of the new-born occurring very soon after birth, often in the most robust of infants, comes on without any apparent focus of infection. From analogy, however, we know that some of the severest types of septicemia or toxemia have no visible focus of infection, and as these hemorrhages usually resemble an acute infection in their sudden onset, with restlessness, loss of appetite and temperature (often only in the early stages), we have come clinically to believe in the infectious origin of this condition. In support of this there are on record a few positive blood cultures, but from this standpoint it has neither been studied carefully enough nor in a sufficiently large number of cases under the best conditions to be able to assert anything very definite. There can be no question that certain of these cases are the result of direct trauma, for at this period the blood vessels are in a most fiiable state, but, disregarding these few, the great number, certainly, from our clinical knowledge of the process must be considered as infectious, and to uphold this theory we have many known infections that do cause more or less hemorrhage in one stage or another of their invasion to use as analogies. The hemorrhage that occurs a little later during the appearance of idiopathic jaundice has an entirely different explanation. As this jaundice is a physiological process, so the hemorrhages found in combination with it are chemico-physiological in their occurrence. And as the one subsides, the other disappears for the same reason. There will be an attempt later to show the part the liver plays in these hemorrhagic conditions. Suffice it to say here that, as is well known, in the presence of jaundice the tendency to bleed is often manifest and the stopping of these hemorrhages is quite a difficult process, not uncommonly impossible.

Still later we find a much larger group of cases which are at present unadvisedly found under several headings with the common prefix "purpura." That this is one condition with different manifestations is far more probable than the current idea of supposing each manifestation an entity, with hemorrhages as a symptom. It is perfectly true that we find this hemorrhagic condition appears as a secondary process, but even so, there is no reason to suppose that etiologically it is identical with true purpura. For instance, 
there is certainly no connection between the hemorrhages that occur in epidemic cerebrospinal meningitis and those found in what is termed purpura hemorrhagica, one being a purely toxic condition, while the other, though perhaps toxic in origin, yet has a definite blood picture, which accounts for the hemorrhages. Taking into account all purpuras, those found in conjunction with some definitely known disease, such as scarlet fever, smallpox, measles, diphtheria, etc., or secondary purpuras as they are called, really form a very small class compared to the primary purpuras which have the same etiological basis though having a variety of manifestations, sometimes all the manifestations appearing in a case, sometimes only one. Just what the primary causal factor in these conditions is, no one is at present ready to state, but it has been ascertained by very careful investigation that in these primary conditions there is a marked diminution in one of the cellular elements of the blood, namely, the platelets, and we shall see later in discussing the experimental work the importance of this finding.

In considering the congenital or hereditary conditions in which spontaneous hemorrhages occur, the alterations are found to be materially different from those in primary hemorrhagic conditions. The term " hemophilia" has been applied to these congenital or hereditary hemorrhagic states, and there are a number of theories as to their origin. Immerman's theory of a congenital disproportion between the volume of blood and the size of blood vessels leading to increased blood pressure cannot be upheld under the light of experimental evidence, which has shown that the blood pressure in a majority of cases is below normal. Sahli has suggested the theory that there is a chemical alteration in the walls of the blood vessels producing a functional disturbance rather than any morphological change, and according to this theory hemorrhages would be due to increased osmosis rather than to any change in the factors involved in coagulation. The coagulation time in these conditions is at present under dispute. In conjunction with Sahli's theory, it is claimed further that there is a diminution in some cases amounting to total absence of one of the precursors of thrombin, namely, thrombokinase. This alteration is supposed to be a functional one in the body cells, transmitted in a hereditary line through the germ plasm. This theory seems to be the one which answers best the changes which we find in this congenital condition and gives a clue to why treatment has been only of very temporary value.

The hemorrhages that appear in the course of certain infections or those which are due to an infectious origin primarily, as is the hemorrhage of the newborn in certain cases, are due not to the presence of the organism, but to the production of toxins within the body which act chemically either as inhibitory agents on coagulation or by locking up in a loose chemical union the available normal factors that enter into coagulation. These toxins chemically are complex proteid substances, such as peptone and albumoses. Ex- perimentally it is possible to reproduce conditions similar to these infectious hemorrhages by the intravenous injection of these same toxins or by other peptones or albumoses, as can be done by the injection of hirudin or leech extract. The toxic element of snake venom is composed of this same form of chemical poison, though the action of the venom is much more rapid, and more toxic, and goes on to a further degree to active hemolysis or laking of the blood. We are all more or less familiar with the rapidly fatal cases of measles and diphtheria, popularly termed black measles and black diphtheria. In these conditions a similar process takes place and the toxic agents belong to the same group of albumoses. These changes in the blood make it more fluid and so allow osmosis to take place more readily. Further, these poisons act on the endothelium of the blood vessels, making them more permeable. This condition of endotheliolysis has been described and pointed out pathologically as well as experimentally. We can see from this description why most specific treatment has been useless, for the rôle that a specific would have to play would be to enter into a more stable union with the toxin than the elements of the blood were able to, or in some way to intercept or neutralize the effect of the toxin, or to aid in the rapid elimination or destruction of the toxin. We can understand why the general supportive or eliminative lines of treatment have always held such an important place in these conditions, for if the organism is supported long enough, it has the power within itself of producing substances which overcome or neutralize these toxic substances and also of inducing the elimination of them. In this we find the most promising field for the production experimentally of specific neutralizing or immunizing substances, as has been done in the antiserum for snake venom.

In the primary purpuras we find, as has been stated, a diminution of one of the precursors of thrombin, the prothrombin. It has been proved both clinically and experimentally that prothrombin is derived from the blood platelets. In these purpuras we find a very marked diminution of the platelets, as low as 7,000 when the normal count averages 500,000 to the cubic millimeter. So with this definite and constant blood picture, it is fair to assume that to their absence the hemorrhagic condition is due. It has been shown that when the platelets are removed, or, in other words, when the prothrombin is absent, the coagulation time is not affected, but only the contractility of the clot, so that instead of getting a firm, normal clot with the serum separating, we get a gelatinous homogeneous mass with little or no separation of fibrin and serum. This is exactly the condition we find in purpura. The coagulation time is within normal limits. Very often a short coagulation time is found, but the form of the clot is materially changed and, instead of a firm clot with the separation of serum, we get a gelatinous one with little or no tendency for the serum to separate. Just what causes the diminution of the platelets is still under consideration. 
The French school, which is practically the only school which has done any work along this line, think that two factors are necessary for the production of this condition. They claim to have produced conditions exactly similar to purpura by first causing certain changes in the liver, which consist mainly in a form of slow necrosis. When this is accomplished, the central nervous system is intoxicated by the intradural injection of certain toxic substances, such as blood serum from grave hemorrhagic cases or by diphtheria toxin. Apparently there is little doubt that they have and are able to produce hemorrhagic conditions by these means, but whether they are exactly similar to primary purpura has not been as clearly demonstrated, for they have not shown that in this way they can diminish the number or affect the output of the platelets.

From a clinical standpoint, however, these experiments are most suggestive and explain the appearance of hemorrhagic conditions which occur during the course of certain liver changes or affections. The tendency to bleed in jaundice has already been referred to, and we are not surprised to learn that experimental intravenous injection of bile or the introduction of bile salts, the taurocholate or glycocholate of soda, is capable of producing a condition of incoagulability of the blood. In liver necrosis from chloroform poisoning this same condition of incoagulability of the blood is found. Whether this is a chemical combination of the bile salts and the calcium of the blood has not yet been ascertained, but this seems the most likely explanation. If so, then the work of the French school could be explained on this ground rather than on the supposition that a true purpura is produced.

I would offer this explanation for a certain number of cases of hemorrhage of the newborn, for it is well known how often liver lesions are found in infants and how easily liver lesions are produced in infants; also how unstable their central nervous systems are; so that such a combination of disturbances, if the former alone does not offer sufficient reason, would give certainly a very plausible explanation for these early hemorrhages, especially when infections can be definitely ruled out, as appears to be the case in a certain proportion. This explanation, I believe, has never been offered, but I can see no objection to its validity.

In the congenital or hereditary cases we are still further in the dark. What has been already said about the absence of the tissue coagulin in these conditions is about the limit of our knowledge. That this change is a functional one on the part of all the body cells which normally have a part in the production of this tissue coagulin, and not a chemically or pathologically demonstrable one, is pretty generally accepted. Because of its being a functional alteration of all the cells, it is the most unapproachable, both from an experimental and from a rational therapeutic standpoint. The most that can be hoped for from treatment is the temporary furnishing of the deficiency in tissue coagulin.
Therapeutic measures viewed in the light of experimental evidence must supply the deficiency in each case, or correct faulty performances of normal processes, or act as stimulants for the production of such substances by the body cells. At least they must act as temporary aids to bridge over periods when certain factors are reduced in amount which in time would become normal if protected during the presence of certain foreign substances.

One of the most used means in such conditions is the administration of salts, principally calcium, either by mouth or subcutaneously. From testtube experiments it can be easily demonstrated that the addition of calcium to blood plasma rendered incoagulable by various means does increase the coagulability, but when we come to testing its value when given to a patient, it has been very clearly shown that though the ionizable calcium salts of the blood, and this is the only form of calcium that enters into the coagulation of the blood, can be slightly increased, yet the possible increase is so slight that the coagulability is not affected in the least. This is tested by precipitating out all the ionizable calcium crystals and counting them with the ordinary Thoma-Zeiss apparatus used for blood counts and comparing the coagulation time before and after giving the calcium. It has been found that the ingestion of $125 \mathrm{gm}$. of calcium does not raise the coagulability of the blood in the least, so that no matter what doses of calcium we give, in reality we are not accomplishing the purpose for which we intended this remedial agent, that is, increasing the coagulability of the blood.

The use of gelatin is entirely empirical, and, as far as I can learn, it is inert in its power to increase the coagulability of the blood either in vitro or in vivo.

Adrenalin as usually given is also inert, for adrenalin solution given subcutaneously does not get into the circulation before it has lost its power. This can be very easily demonstrated experimentally by watching the difference in effect on the blood pressure of subcutaneous and intravenous injections. Subcutaneously there is no effect on the blood pressure, or it is too slight to be of any value. Locally it is of value as a styptic and, if given intravenously and in sufficient amount and strength, it has been shown to have a marked temporary effect which, however, wears off very readily, so that its value is limited to such cases as call for only a temporary measure to tide over some critical period that will be of very short duration. In the intravenous injection of adrenalin, we get an increased blood pressure which is, of itself, not to be desired, and which might increase rather than decrease the hemorrhagic condition for which the adrenalin has been administered. Its action is entirely a mechanical one on the blood vessels, making osmosis more difficult by increasing the contractile tone of the blood vessels, more especially the peripheral vessels and capillaries.

In the past few years, since the revival under improved technic of blood transfusion, we have 
really gained the most rational means of combating these conditions, for by supplying fresh blood we supply all the elements necessary for the actual increase in the coagulability of the blood. We supply all the factors that enter into coagulation from a perfectly normal subject. The process, however, is not an easy one to accomplish, and is one that requires very careful and especial technic. The men that have been most successful in this procedure are the men who have done the most experimental operating, as time and familiarity with each step in the procedure are most important. The procedure has some dangers aside from the surgical risks, the main danger lying in the possibility that the donor's blood may cause hemolysis or laking of the recipient's or patient's blood. Tests for this should be made in all cases where this is feared. This danger is minimized if the blood is taken from a member of the patient's family.

However, not all cases are severe enough to indicate or warrant such radical measures, and though unquestionably the best and most satisfactory method in severe cases, a substitite for the less severe cases and ones which do not really endanger the lives of the patients is very desirable. In this connection the injection of fresh animal serum which contains all the ferments and salts that are found in the blood itself - in fact, there is more ferment both in amount and in potency than in the blood before it has clotted - has been tried. Experimentally almost every known serum has been used, and the ones found most efficacious are holse serum, rabbit serum and human serum. Horse serum is the least capable of producing the desired changes. Rabbit serum is appreciably more potent, and human serum is, of course, the best when it can be procured, which is seldom.

I have been carrying on some experimental work along these lines, comparing the reactivating power of different animal sera, and have found, as might be supposed, that this power varies somewhat with the different sera. This power of reactivating, or, in other words, the ability to cause coagulation by adding a small amount of serum to a blood that has artificially been made incoagulable, resides in a substance which is thermolabile and can be destroyed by heating to $56^{\circ} \mathrm{C}$. Therefore, most probably it is the fibrin ferment of the thrombin that is the real factor and plays the important rôle in producing these changes. The power of reactivation is not lost for a considerable period, for I have found sera as active at the end of forty days as when first drawn, though at the end of one hundred days, in one instance, it had disappeared. Rabbit serum is very easily procured by tapping the heart directly with an ordinary needle and syringe and drawing off some 5 to $10 \mathrm{ccm}$. of blood from each rabbit. Rabbits can be kept for quite a period, bleeding them about every ten days or two weeks.

The carotids may be tapped by means of the Pasteur carula test-tube, which can be blown as desired. This is the way in which I have found it most convenient to procure the serum. The advantages of this are that a sufficient amount of serum can be procured from one rabbit, that in any one dose there is no admixture of different sera, and that the asepsis can be more carefully carried out. I have often obtained as high as $100 \mathrm{ccm}$. of blood from a single rabbit, and this furnishes about $50 \mathrm{ccm}$. of serum. The dose of this serum runs from 10 or $15 \mathrm{ccm}$. in a mild case or a very young infant to 30 or $50 \mathrm{ccm}$. in a more severe case or an older child. In severe cases this dose can be repeated at four to six-hour intervals; in less severe cases it is given once a day for several days or until no new hemorrhages have appeared for about twenty-four hours; sometimes only one or two doses are necessary to control quite severe bleeding.

The cases in which it is most indicated, of course, are those deficient in some one of the fibrin ferment precursors or in conditions in which these are unable to act, that is, in primary purpura where the prothrombin is absent, in jaundice where the proferments are not able to act, and to a markedly less degree it is of value in the hemophiliac cases of the congenital type where there is a functional change. In these cases the serum is able to furnish only temporarily a deficiency for a period of a few days or weeks, in some cases lasting even one or two years, but it cannot be permanent, for the primary functional derangement still continues, and when the power of the serum is spent, or the effect of its stimulation worn off, then the condition recurs, while in the two first-mentioned conditions its effect is permanent if successful, for the trouble is only a temporary one, and by the time the ferment supplied has disappeared, the original condition that called for its use has also disappeared.

I have had opportunity of trying this method in only a few cases during the past year and a half at the Children's Hospital, with results which I think are quite favorable to its further use. I have tried it in three cases of purpura simplex, two cases of purpura hemorrhagica, one case of hemorrhage in the newborn and in two cases of congenital hemophilia.

The treatment in these cases was successful in all but one, which, as the autopsy report showed, was probably not a true purpura, but a secondary purpura in acute miliary tuberculosis, and although the hemorrhages in this case were controlled for a sufficient time before death to show the value of the treatment, yet the primary trouble, of course, was not stayed.

\section{THE TREATMENT OF SUMMER DIARRHEA AS INFLUENCED BY ETIOLOGY.}

BY CHARLES HUNTER DUNN, M.D.,

Clinical Instructor in Pediatrics, Harvard Medical School; Assistant Physician at the Children's Hospital; Assistant Physician and

Additions to the knowledge of diseased conditions are constantly being made, and such additional knowledge, especially on the etiologic side, usually carries with it suggestions for bettering our methods of treating these conditions. These 